\title{
Identification of exon skipping events associated with Alzheimer's disease in the human hippocampus
}

\author{
Seonggyun Han', Jason E. Miller², Seyoun Byun', Dokyoon Kim²,3, Shannon L. Risacher ${ }^{4}$, Andrew J. Saykin ${ }^{4,5}$,
} Younghee Lee ${ }^{1 *+}$, Kwangsik Nho $\mathrm{N}^{45^{*+}}$ and for Alzheimer's Disease Neuroimaging Initiative

From The International Conference on Intelligent Biology and Medicine (ICIBM) 2018

Los Angeles, CA, USA. 10-12 June 2018

\begin{abstract}
Background: At least $90 \%$ of human genes are alternatively spliced. Alternative splicing has an important function regulating gene expression and miss-splicing can contribute to risk for human diseases, including Alzheimer's disease (AD).

Methods: We developed a splicing decision model as a molecular mechanism to identify functional exon skipping events and genetic variation affecting alternative splicing on a genome-wide scale by integrating genomics, transcriptomics, and neuroimaging data in a systems biology approach. In this study, we analyzed RNA-Seq data of hippocampus brain tissue from Alzheimer's disease (AD; $n=24)$ and cognitively normal elderly controls $(C N$; $n=50$ ) and identified three exon skipping events in two genes (RELN and NOS1) as significantly associated with AD (corrected $p$-value $<0.05$ and fold change $>1.5$ ). Next, we identified single-nucleotide polymorphisms (SNPs) affecting exon skipping events using the splicing decision model and then performed an association analysis of SNPS potentially affecting three exon skipping events with a global cortical measure of amyloid- $\beta$ deposition measured by $\left[{ }^{18} \mathrm{~F}\right]$ Florbetapir position emission tomography (PET) scan as an AD-related quantitative phenotype. A whole-brain voxel-based analysis was also performed.
\end{abstract}

Results: Two exons in RELN and one exon in NOS1 showed significantly lower expression levels in the AD participants compared to CN participants, suggesting that the exons tend to be skipped more in AD. We also showed the loss of the core protein structure due to the skipped exons using the protein 3D structure analysis. The targeted SNP-based association analysis identified one intronic SNP (rs362771) adjacent to the skipped exon 24 in RELN as significantly associated with cortical amyloid- $\beta$ levels (corrected $p$-value $<0.05$ ). This SNP is within the splicing regulatory element, i. e., intronic splicing enhancer. The minor allele of rs362771 conferred decreases in cortical amyloid- $\beta$ levels in the right temporal and bilateral parietal lobes.

(Continued on next page)

\footnotetext{
*Correspondence: younghee.lee@utah.edu; knho@iupui.edu

${ }^{\dagger}$ Younghee Lee and Kwangsik Nho contributed equally to this work.

'Department of Biomedical Informatics, University of Utah School of

Medicine, Salt Lake City, UT, USA

${ }^{4}$ Center for Neuroimaging, Department of Radiology and Imaging Sciences

and Indiana Alzheimer Disease Center, Indiana University School of Medicine,

Indianapolis, IN, USA

Full list of author information is available at the end of the article
}

(c) The Author(s). 2019 Open Access This article is distributed under the terms of the Creative Commons Attribution 4.0 International License (http://creativecommons.org/licenses/by/4.0/), which permits unrestricted use, distribution, and reproduction in any medium, provided you give appropriate credit to the original author(s) and the source, provide a link to the Creative Commons license, and indicate if changes were made. The Creative Commons Public Domain Dedication waiver (http://creativecommons.org/publicdomain/zero/1.0/) applies to the data made available in this article, unless otherwise stated. 
(Continued from previous page)

Conclusions: Our results suggest that exon skipping events and splicing-affecting SNPs in the human hippocampus may contribute to AD pathogenesis. Integration of multiple omics and neuroimaging data provides insights into possible mechanisms underlying AD pathophysiology through exon skipping and may help identify novel therapeutic targets.

Keywords: Alzheimer's disease, Exon skipping, RNA-sequencing, RELN, NOS1, Neuroimaging, Human hippocampus

\section{Background}

Alzheimer's disease (AD) is a progressive neurodegenerative disorder pathologically characterized by an accumulation of both toxic amyloid- $\beta$ plaques and neurofibrillary tau tangles in the brain [1]. Twin studies as well as more recent large-scale genome-wide association studies (GWAS) have demonstrated that genetic susceptibility factors play an important role in the development of the AD, although there is still a substantial portion of missing heritability to be identified [2, 3]. Increasing evidence suggests that widespread transcriptional changes accompany the onset and progression of $\mathrm{AD}$ [4-8]. In particular, the aberration in the control of gene expression by alternative splicing is implicated in AD [9-12]. Previous whole transcriptome sequencing analyses revealed gene expression and alternative splicing changes in the AD-affected brain regions [4, 1012]. Several alternatively spliced $A D$ candidate genes such as $C L U$ and CD33 were reported to be associated with AD pathogenesis [13, 14]. Thus, it could provide valuable information on the underlying pathology associated with the AD to identify other alternative spliced genes and $\mathrm{AD}$-associated single-nucleotide polymorphisms (SNPs) affecting splicing regulation.

Alternative splicing is the process by which a single gene can produce multiple RNA isoforms through the splicing in and out of different portions of the transcript. Although it is an important mechanism for increasing biological complexity through generating tissue-specific transcript, miss-splicing can lead to different disease states. For example, in humans, more than $90 \%$ of genes are alternatively spliced [15] and generating 100, 000 proteins through different usage of exons (i.e. alternately spliced exons) [16]. Furthermore, such transcripts or certain exon skipping are expressed in the tissue- and disease-specific manner. Especially more genes are alternatively spliced in the brain than other tissues [16], and specific exons are brain-specially skipped or included in AD-associated genes including APP [13, 16], PSEN1 [17], PSEN2 [17], APOE [16], and MAPT [18-20].

SRE is an ancillary cis-acting element as a part of the splicing machinery that assists a spliceosome to correctly recognize the exon-intron boundary by recruiting activator or repressor Trans-acting RNA-binding proteins (RBP) [21]. There are four types of SREs, exonic splicing enhancers (ESEs), exonic splicing silencers (ESSs), intronic splicing enhancers (ISEs), and intronic splicing silencers (ISSs). Mutation in any sites of SREs changes the binding accuracy of spliceosome to the splice sites and potentially result in the aberrant exon skipping events producing disease-causing proteins. Furthermore, 15\% of diseasecausing mutation is estimated to be associated with splicing including SREs and splice sites [22-24], and we have previously demonstrated that alternative splicing is useful for identifying disease-associated variation in the human genome $[25,26]$. It remains difficult to identify novel genes and molecular mechanisms associated with splicing that underlie $\mathrm{AD}$ pathological hallmarks due to the nature of studying the brain and neuropathological traits.

This study explores how transcriptomics, genomics and neuroimaging endo-phenotypes can be leveraged as a means to clarify our understanding of the genetic architecture of AD. Using RNA-Seq data from cognitively normal elderly controls and AD-affected human hippocampal tissues, alternative splicing isoforms were evaluated by measuring exon skipping. A computational pipeline to identify exon skipping events using RNA-Seq data and a splicing decision model to identify actionable loci among common SNPs for gene regulation were applied in this study to gain insights into the functionality of the variations and emphasized their importance for the AD pathology [27]. We identified SNPs affecting exon skipping by analyzing sequence-driven alternative splicing models and by scanning the genome for the regions with putative splicing regulatory elements (SREs) motifs [26, 27]. Aberrant alternative splicing sites were detected that associate with the AD using SNPs within regions affecting the exon skipping as associated with AD-related neuroimaging biomarkers, a global cortical measure of amyloid- $\beta$ deposition measured by $\left[{ }^{18} \mathrm{~F}\right]$ Florbetapir position emission tomography (PET) scans. These results provide a new link between alternative splicing changes and AD.

\section{Methods}

\section{Study sample and RNA-sequencing data analysis}

RNA-Seq data (bam files) were downloaded from the Allen Brain Atlas (http://human.brain-map.org/). RNA was isolated from the hippocampus tissue of brains of $\mathrm{AD}$ patients (AD; $n=24)$ and non-AD elderly controls $(\mathrm{CN} ; n=50)$ from the Adult Changes in Thought (ACT) study. The ACT study is a longitudinal population-based 
prospective cohort study of brain aging and incident dementia in the Seattle metropolitan area, as described in detail in previous studies [28, 29]. RNA sequencing was performed using an Illumina HiSeq 2500 with v4 chemistry, producing a minimum of 30 M 50 bp paired-end clusters per sample. Raw read files (bam files) were aligned to the GRCh38 reference genome, as described in detail (http://aging.brain-map.org/). The average reads of all individual participants $(n=74)$ were $55,015,989$.

\section{Identification of exon skipping events}

A bioinformatics splicing decision model based on our previous study [27] was implemented to identify exon skipping events and genetic variation affecting exon skipping using RNA-Seq data on a genome-wide scale in the human hippocampus (Fig. 1). First, to estimate the normalized usage for each exon, total reads for each exon were counted from RNA-seq data (bam files with the mapped reads) using HTSeq [30, 31] and there were only exon reads, but no junction reads. Junction reads are crucial information to know which exon structure is changed. However, as we obtained the exon reads from the bam files, we run DEXSeq to measure exon expression level that basically uses exon reads only [31]. Next, expressed exons (exon skipping events) between $\mathrm{AD}$ and $\mathrm{CN}$ were identified using a generalized linear regression model in DEXSeq. The statistical cutoff as a significantly differentially expressed exon (exon skipping) was false discovery rate (FDR)-corrected $p$-value $<0.05$ and fold change $>1.5$, which was calculated with p.adjust function in R, i.e., correction for multiple comparisons was performed using the FDR-based multiple comparison adjustment with the Benjamini-Hochberg procedure at a 0.05 level of significance.

\section{Identification of SNPs in splicing regulatory elements associated with exon skipping events}

We have developed a splicing decision model for identifying SNPs affecting splicing regulatory elements (SREs) with exon skipping by using alignment information for four alternative splicing datasets from the UCSC genome browser: mRNAs from GenBank [32], Ensembl Gene Predictions [33], AceView Gene Models [34], and UCSC known genes [35] and a set of predicted hexameric SRE motifs, as described in detail in previous publications $[26,27]$. We searched for all potential SRE sites that are perfectly matched with any of these hexamers in intragenic regions (exons and introns). Our study included three types of SREs available for this time, ESE, ESS, and ISE according to its location and function without ISS as the data of ISS hexameric sequences are not available. We then compiled genotype data and SRE regions with skipping of the adjacent exon to intron or skipping of the exon embedding SRE region, which is a definition of splicing decision model that computationally predict the

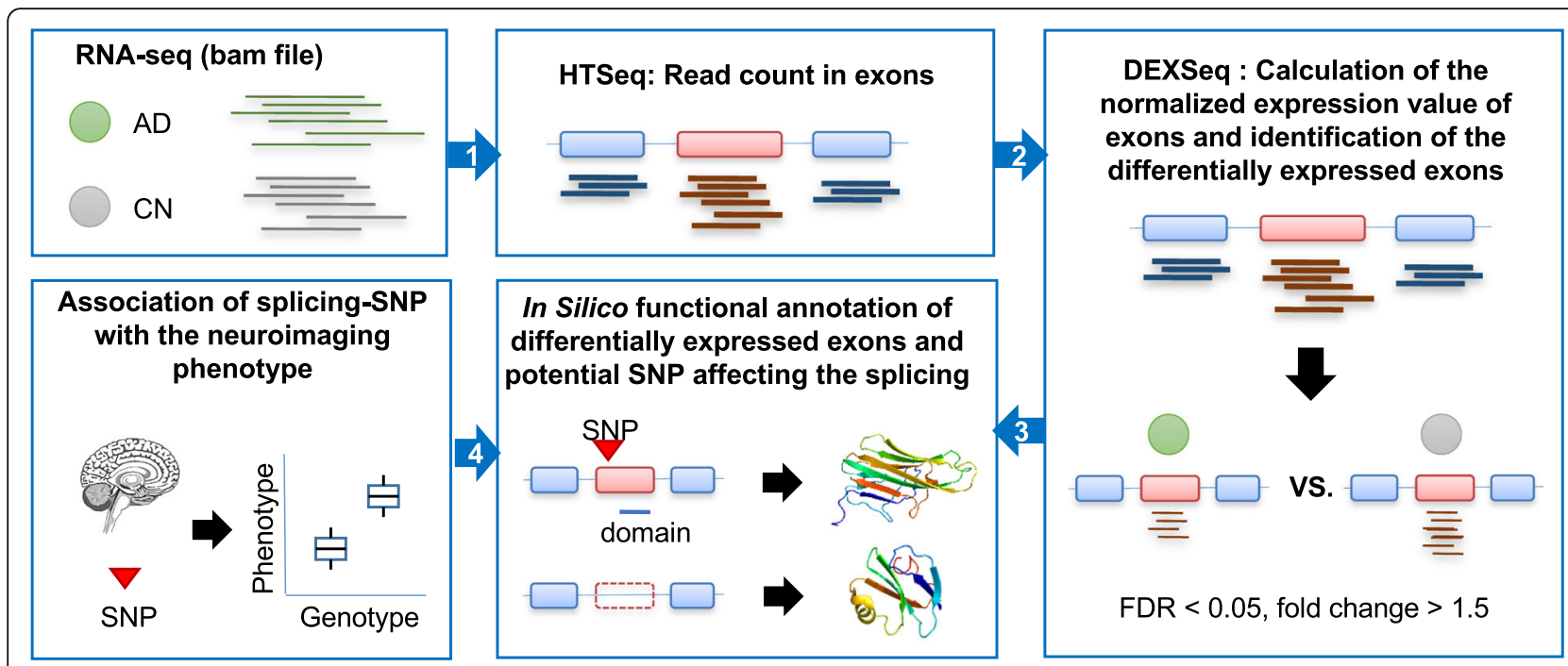

Application to AD: RNA-seq data of hippocampus samples with AD and CN from Allen Brain Atlas

24 AD cases

$50 \mathrm{CN}$ cases
Identification of two known genes

in $A D$ with potential novel exon skipping (RELN and NOS1)
Modulated exon usage in RELN and NOS1, and the splicing-SNP (potentially affecting splicing) associated with neuroimaging phenotype

Fig. 1 Computational pipeline for identifying AD-associated exon skipping events and SNPs affecting exon skipping 
loss-of-function of SRE by SNP. Using the splicing decision model, we identified SNPs within SREs associated with exon skipping events.

\section{Functional annotation of differentially expressed exons}

The impact of exon skipping events on protein structure and function was evaluated in silico. The skipped exons are translated into a functional domain, lead to out of frame through a frameshift, and change their corresponding protein structure using UniProt web browser and RaptorX [36].

\section{Neuroimaging and genotyping analysis}

$\left[{ }^{18} \mathrm{~F}\right]$ Florbetapir PET scans downloaded from the Alzheimer's Disease Neuroimaging Initiative (ADNI) were pre-processed as described [37]. $\left[{ }^{18} \mathrm{~F}\right]$ Florbetapir PET scans were intensity normalized by the whole cerebellum. The normalization yielded standardized uptake value ratio (SUVR) images. The ongoing ADNI study was launched in 2003 to test whether serial magnetic resonance imaging (MRI), PET, other biological markers, and clinical and neuropsychological assessment could be combined to measure the progression of MCI (mild cognitive impairment) and early $\mathrm{AD}$, as published previously [38, 39] and found at www.adni-info.org. Genotyping for ADNI was performed using three different Illumina genotyping platforms. We imputed un-genotyped SNPs separately in each platform using $\mathrm{MACH}$ and the HRC (Haplotype Reference Consortium) data as a reference panel after standard sample and SNP quality control procedures and selection of only non-Hispanic Caucasian participants [40].

\section{Association of SNPs affecting exon skipping with AD- related neuroimaging endophenotype}

First, an association analysis was performed for SNPs that potentially affect identified exon skipping events with a global cortical measure of amyloid- $\beta$ deposition as measured by PET scans in an AD-related quantitative phenotype. The global cortical amyloid- $\beta$ level was calculated as a mean regional SUVR value extracted for the frontal, parietal, temporal, limbic, and occipital lobes using the MarsBaR toolbox implemented in the Statistical Parametric Mapping 8 (SPM8) software (http:// www.fil.ion.ucl.ac.uk/spm/software/spm8/) [41]. Also, a detailed whole brain-based neuroimaging analysis was performed using multivariate models for amyloid- $\beta$ levels on voxel-by-voxel bases. Age at baseline, sex, and years of education were used as covariates for the association test. Correction for multiple comparisons was performed using false discovery rate (FDR) correction method at a 0.05 level of significance.

\section{Results}

This study analyzed RNA-Seq data of hippocampus brain tissues from 74 participants from the ACT study. Participants were diagnosed as cognitively normal elder controls $(\mathrm{CN})$ or AD patients. As shown in Table 1, there were $24 \mathrm{AD}$ and $50 \mathrm{CN}$ participants, and the mean Braak stages of $\mathrm{AD}$ and $\mathrm{CN}$ were 4.4 and 2.78 , respectively.

\section{Differentially expressed exons in AD hippocampus tissue} Using the computational pipeline described in Fig. 1, normalized expression levels were calculated for each exon in a genome-wide manner, and a generalized linear regression model was used to identify $\mathrm{AD}$-associated exon skipping events. After adjusting for multiple comparisons using the FDR method, we identified three exon skipping events in two genes, RELN and NOS1, as significantly associated with the AD (FDR-corrected $p$-value $<0.05$ and fold change $>1.5$ ). Two exons in RELN, exons 24 and 37 , showed significantly lower expression levels in $\mathrm{AD}$ patients compared to $\mathrm{CN}$ participants, suggesting that the exons tend to be skipped more in the AD (Fig. 2a). The exon 24 was predicted to encode a hEGF domain region (PF00008, Fig. 2b). Furthermore, for $\mathrm{AD}$ participants, we investigated whether the Braak stage is associated with the exon skipping event. In $24 \mathrm{AD}$ participants, 19 were in the Braak stages 4, 5 and 6. Among the $19 \mathrm{AD}$ participants in higher Braak stages, 15 showed significantly lower expression levels of the exon 24 compared to the other $4 \mathrm{AD}$ participants (one-way chi-squared test, $p$-value $=0.01$ ), suggesting that the exon tends to be skipped more in higher Braak stages of AD participants. In NOS1, exon 23 had lower expression levels in $\mathrm{AD}$ patients compared to $\mathrm{CN}$ participants (Fig. 3a) and was a part of the $\mathrm{NO}_{-}$synthase domain (PF02898, Fig. 3b). Our results suggest that these exon skipping events may affect the function of the corresponding protein products.

Table 1 Demographic characteristics of 74 study samples

\begin{tabular}{llllll}
\hline & \# of sample & Male/Female & Age & Education & Braak stage \\
& & & {$[$ min-max] } & [min-max] & \\
\hline $\mathrm{AD}$ & 24 & $11 / 13$ & $91(7.2)[78-100+]$ & $14.4(3.25)[6-21]$ & $4.4(1.70)[0-6]$ \\
$\mathrm{CN}$ & 50 & $30 / 20$ & $89(6.9)[78-100+]$ & $14.7(3.07)[8-21]$ & $2.76(1.49)[0-6]$ \\
\hline
\end{tabular}




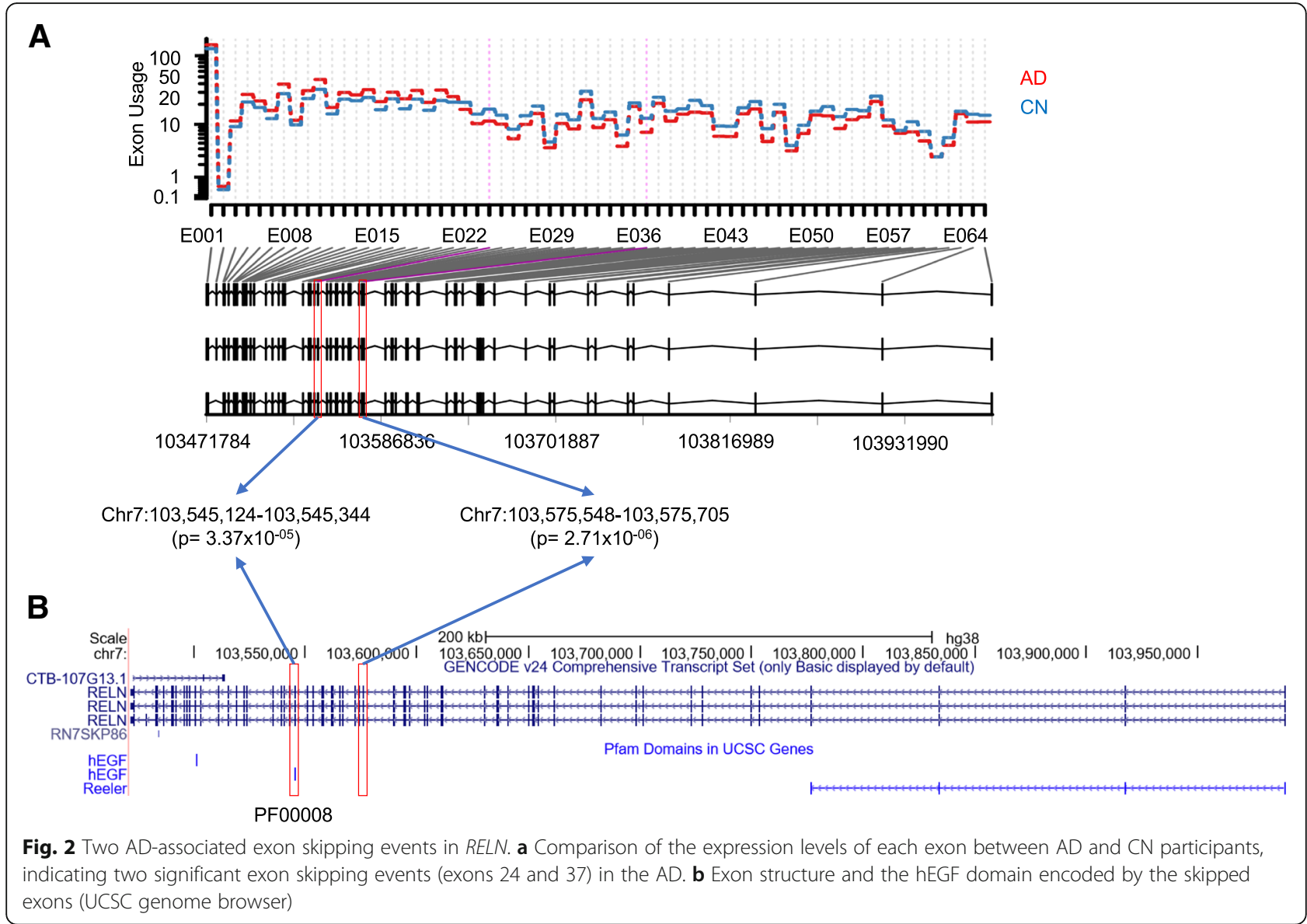

\section{Prediction of the effect of the identified exon skipping events on protein}

To characterize the potential impact of the variants on the protein, each variant was analyzed using UniProt web browser and RaptorX. All of three skipped exons in RELN and NOS1 resulted in producing a coding sequence which is out-of-frame, potentially generating an undesired protein product. As presented in Fig. 4, the first AD-associated exon skipping (exon 24) in RELN showed lower expression levels of the exon in $\mathrm{AD}$ compared to $\mathrm{CN}$ participants (Fig. 4b; FDR-corrected $p$-value $=0.034$, fold change $=1.51$ ). The adjacent exon 23 and 25 showed similar levels of difference but not significant (FDR $=0.154$ for both exons, fold change $=1.39$ and 1.37 , respectively). The transcript (transcript1) retaining the exon 24, which encodes the hEGF, likely results in a functional version of the RELN gene, while the transcript which lacks exon 24 will lose the hEGF domain and thus produce the truncated protein product due to the out-of-frame of the exon length (Fig. 4a). Structural analysis of the truncated version of the protein suggests that exon skipping may be implicated in functional changes of RELN in the AD (Fig. 4c). The other exon (exon 37) in RELN is presented in Additional file 1: Figure S1. Additionally, exon 23 was identified as being skipped in NOS1 (Fig. 5a). AD participants had significantly lower expression levels of the exon compared to CN (Fig. 5b; FDR corrected $p$-value $=0.043$, fold change $=1.90$ ). The transcript (transcript1) retaining the exon 23, which encodes a part of the NO_synthase, can be translated into the protein with normal functions of NOS1. In contrast, the transcript with the skipping of exon 23 may not only lose the NO_synthase domain but also produce the truncated protein due to the out-offrame of the exon length. We also showed the partial loss of the protein structure due to the skipped exon using the protein 3D structure analysis (Fig. 5c).

\section{Association of SNPs affecting exon skipping with AD- related neuroimaging phenotypes}

Next, we performed an association analysis of SNPs affecting exon skipping events with a global cortical measure of amyloid- $\beta$ deposition as an AD-related quantitative phenotype. Using the splicing decision model, we first identified 46 and 11 SNPs in RELN and NOS1, respectively, potentially affecting the identified three exon skipping events (MAF > 1\%) from HRC-based imputed ADNI GWAS data. We identified one SNP (rs362771) in intron adjacent to the skipped exon 24 in RELN as significantly 


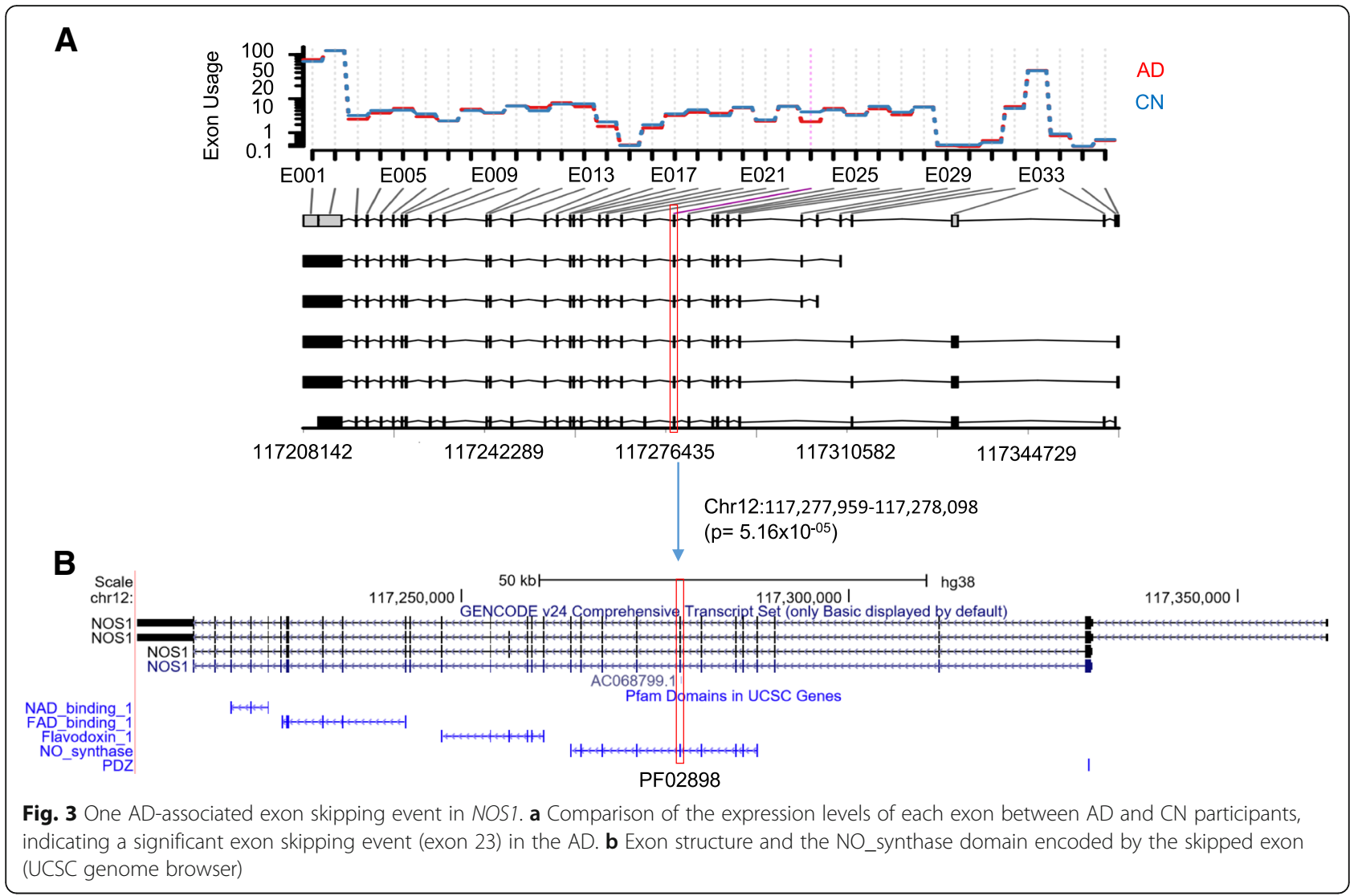

associated with cortical amyloid- $\beta$ levels (Fig. 6a; permutation-based corrected $p$-value $<0.05)$. Furthermore, we performed an unbiased whole-brain-based imaging association analysis using age, sex, years of education as covariates to assess the effect of rs362771 on whole-brain amyloid- $\beta$ deposition and identified significant associations after adjustment for multiple comparisons using cluster-wide FDR procedure. The minor allele of rs362771 conferred decreases in cortical amyloid- $\beta$ levels in the right temporal and bilateral parietal lobes (Fig. 6b). As shown in Fig. 4a, we found that the SNP (rs362771) is located within the ISE site (5th site of hexametric sequence CCTTCC), suggesting that the SNP may affect the exon skipping and the skipped exon may be associated with $\mathrm{AD}$ pathogenesis.

\section{Discussion}

Here we developed a computational pipeline for the identification of exon skipping and a splicing decision model for the identification of SNPs affecting exon skipping. Altered expression patterns (exon skipping events) within two distinct exon regions of RELN and NOS1 in the human hippocampus affected by Alzheimer's disease (AD) were identified. Interestingly, expression levels of identified alternatively spliced exons are decreased in the $\mathrm{AD}$ and the minor allele of one SNP in RELN potentially affecting exon skipping negatively correlate with a global cortical amyloid- $\beta$ burden. Our results indicate that essential exon regions for the RELN and NOS1 genes are alternatively spliced in the AD hippocampus compared to cognitively normal elderly controls. It was previously reported that $R E L N$ delays amyloid- $\beta$ fibril formation and rescues cognitive deficits in an $\mathrm{AD}$ model [42]. Two major neuropathological hallmarks of the $\mathrm{AD}$ are the accumulation of toxic levels of amyloid- $\beta$ molecules and hyper-phosphorylated tau protein that leads to neurofibrillary tangles. Although it was known that RELN and NOS1 were associated with neurologically related traits, the methodology presented here suggests new possible mechanisms by which they may influence AD pathology [43-45].

RELN is an extracellular matrix glycoprotein that plays a number of important roles in the central nervous system (CNS) and its dysfunction is associated with $\mathrm{AD}$ [46-48]. Many studies have found associations between genetic variation in RELN and neurological traits, often using case-control study design [49]. Characterization of these genetic associations is important however, it lacks mechanistic insight that can be provided with interrogating specific regions of the gene, or incorporating other molecular information, such as gene expression. By utilizing a neuroimaging endophenotype, along with transcriptomic information 

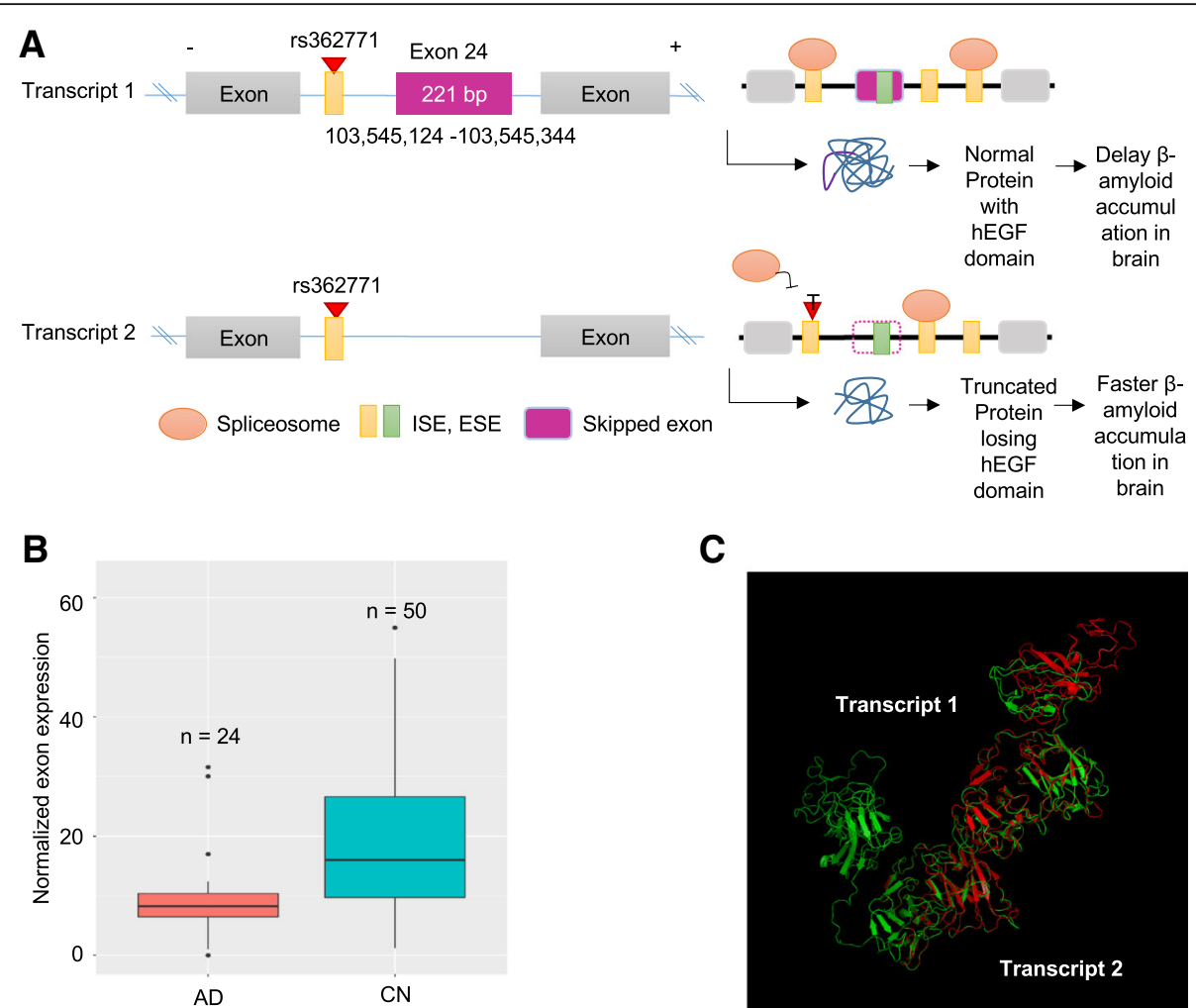

Fig. 4 Functional impact of the AD-associated exon skipping event (exon 24) in RELN. a Schema of the potential functional implication of exon skipping and splicing-associated SNP. b Normalized expression levels for exon 24 between AD and CN participants. c Structure alignment of the pair of transcript1 retaining exon 24 (green) and transcript 2 with the exon skipping (red)

in relation to alternative splicing, we identified differential expression levels among exons in RELN. The function of the $R E L N$ protein is to help mediate cell migration during brain development by activating a signaling pathway through binding of cell surface proteins $[46,50]$. These pathways mediate the process of tau phosphorylation and a reduction of RELN expression can significantly accelerate amyloid- $\beta$ deposition in transgenic AD mice [48]. Thus, differential exonic usage with respect to the $\mathrm{AD}$ may produce an isoform of $R E L N$ protein that potentially accelerates amyloid- $\beta$ deposition and/or mis-regulation of tau phosphorylation, both leading to AD-related phenotypes. Specific exonic expression appears to play an important role. Thus, understanding the role of exon skipping may lead to more consistent conclusions with respect to the role of RELN and other proteins [46].

NOS1, the other gene identified in this work, has also been of significant interest in relation to heart and neuronal function since nitric oxide (NO) is an important signaling molecule that is produced by NO-producing enzymes, such as NOS1 [51]. NOS can produce significant amounts of reactive oxygen species (ROS) that can lead to damaged proteins. Therefore, mis-splicing of NOS1 could lead to increased ROS, and thus increased protein mis-folding and aggregation associated with neurodegenerative diseases (i.e. AD). Both increased and decreased expression of NOS1 have been associated with a cognitive disruption in relation to the $\mathrm{AD}$ [52]. Therefore, it will be interesting to characterize how the expression of different isoforms and exons contributes to the spectrum of phenotypes related to $\mathrm{AD}$ in future work.

Although both genes had previous connections with neuronal development and splicing $[46,51]$, this study makes a novel association between alternative splicing and AD with respect to NOS1 and RELN. Since both RELN and NOS1 could exacerbate different mechanisms by which $\mathrm{AD}$ arises, it would be interesting for future work to investigate the co-occurrence of variants in these genes and how it impacts neurological function. While many advantages of the methodology employed here have been described in detail, it is also valuable to point out some limitations to avoid over-interpretation and reflect on what improvements need to be addressed in future work.

For instance, our sample size was moderate for this study with respect to the expression analysis. Thus, we may have missed some genes with alternative splicing 


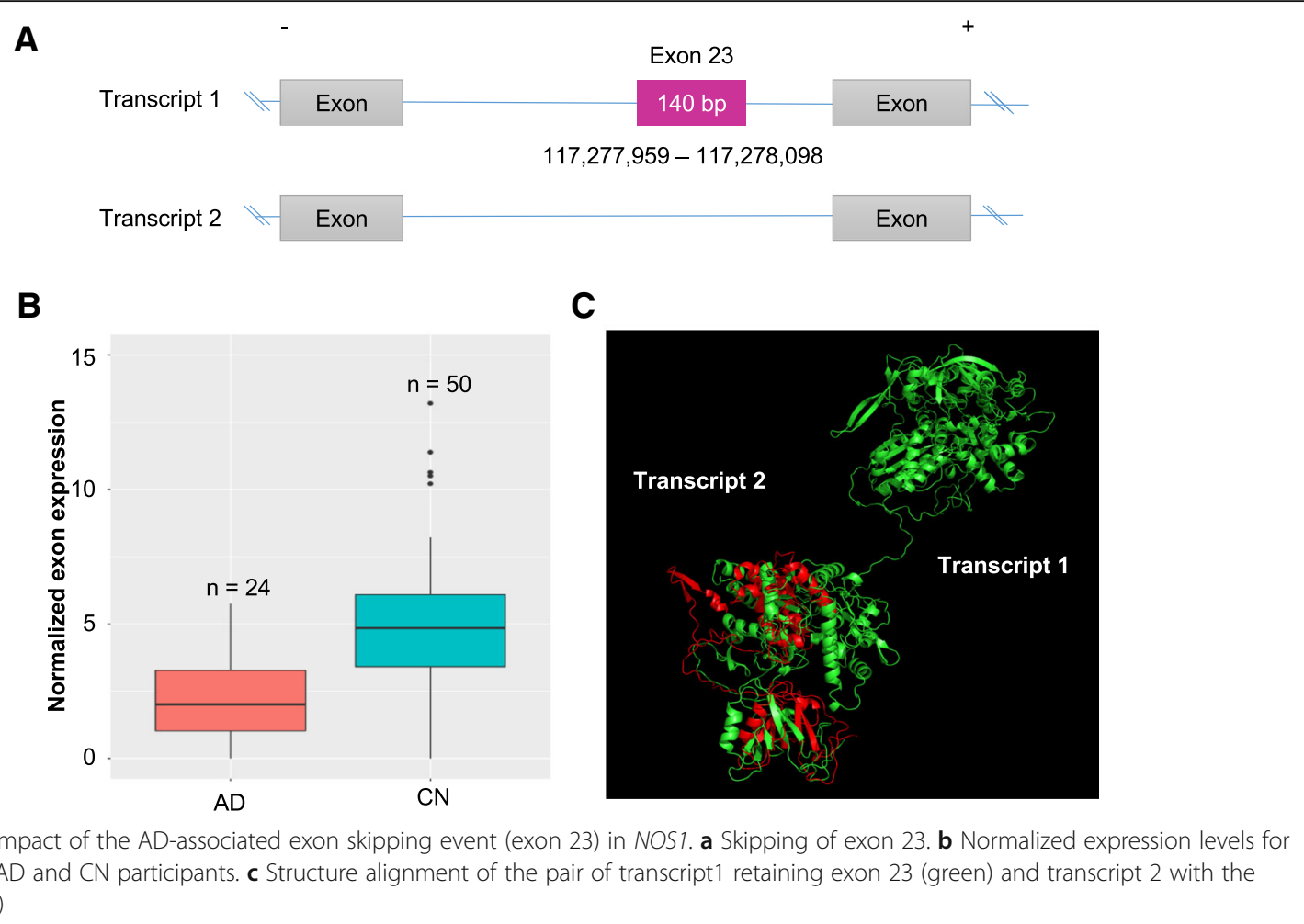

in SRE SNPs associated with AD due to limited detection power. Previous work has suggested sex differences in $\mathrm{AD}$ [47]. The other limitation by the original dataset we used for exon expression quantification is that junction reads were excluded in the downloaded aligned bam file. As junction reads are important resources for estimating more accurate exon skipping events, inclusion of the junction reads may improve the power of our splicing decision model to detect differentially expressed exons in AD. Additionally, it is necessary for follow-up studies to test the function of exon skipping events experimentally as well as to investigate the epigenetic changes influencing the splicing decision - especially association of DNA methylation status in

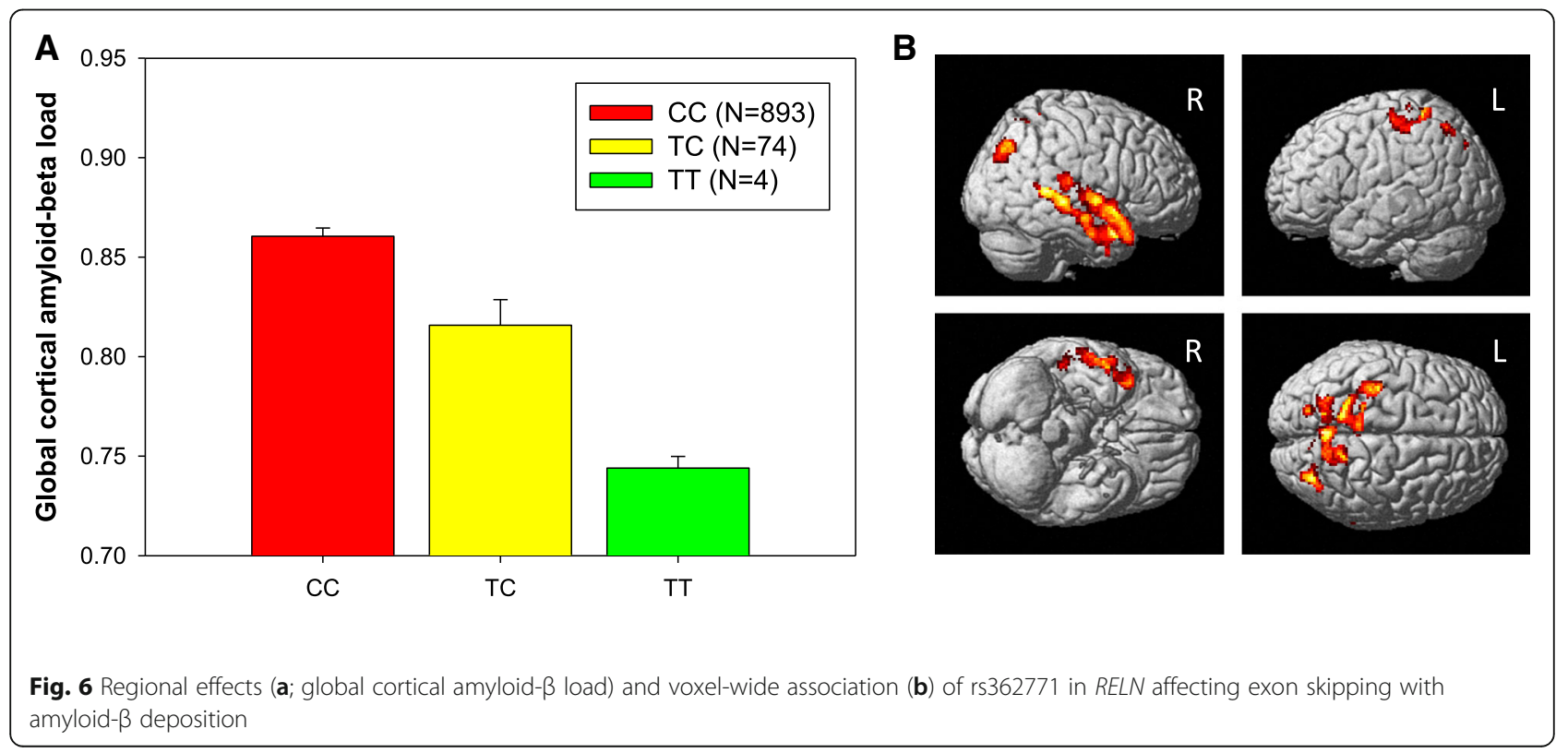


intragenic regions (exons and introns) [53-55]. Finally, here we only explored $\mathrm{AD}$, but the methods applied here could be employed to understand the complex gene-trait relationship among other neurological diseases. In summary, through our integrative analysis of RNA-Seq, genomics, and neuroimaging data, the RELN and NOS1 genes were identified as having differential exon usages with respect to the AD. This work also suggests applying an imaging genetics approach, along with utilizing SRE variants, will help shed light on previously unidentified gene-trait relationships.

Our study was not able to recapitulate a statistically significant exon skipping or spliced isoforms in any of the historical genes (i.e., including APP [13, 16], PSEN1 [17], PSEN2 [17], APOE [16], and MAPT [18-20]). However we observed there were the marginal signals of ten exons in those genes at the unadjusted $p$-value $<0.05$ and fold changes between -1.5 and 1.2, which might be due to the following reasons, 1) alternative splicing events occur in the tissue- and cell type- specific manner and are even more specific to brain regions $[15,16,56,57], 2)$ our study analyzed the RNA-Seq data from the hippocampus regions whereas the known alternative splicing events in the historical genes were found in the whole brain or cerebral cortex region, and 3) as mentioned above, we might miss a signal of those genes under the lack of detection power $(\mathrm{AD}=24$ and non- $\mathrm{AD}$ elderly controls $=50)$.

\section{Conclusion}

In conclusion, this study provides evidence that our novel approach can identify significant exon skipping events and genetic variation potentially affecting exon skipping in Alzheimer's disease. Between RELN and NOS1, three exons are altered in their expressions in the human hippocampus affected by Alzheimer's disease. It also suggests that the functional relationship between exon skipping and protein structures for RELN and NOS1 may be altered in AD pathology. Further studies are needed to better understand the functional role that identified exon skipping and SNPs affecting exon skipping play in AD pathophysiology. Integration of multiple omics and neuro imaging data in a systems biology approach will provide valuable insights into a possible mechanism underlying $\mathrm{AD}$ pathology through exon skipping, thus potentially helping identify novel therapeutic targets.

\section{Additional file}

Additional file 1: Figure S1. Functional impact of the AD-associated exon skipping event (exon 37) in RELN. (A) Schema of the potential functional implication of exon skipping and splicing-associated SNP. (B) Normalized expression levels for exon 37 between AD and CN participants. (C) Structure alignment of the pair of transcript 1 retaining exon 37 (green) and transcript 2 with the exon skipping (red). (PDF 58 kb)

\section{Abbreviations}

AD: Alzheimer's disease; CN: Control normal; ESEs: Exonic splicing enhancers; ESSs: Exonic splicing silencers; FDR: False discovery rate; hEGF: Human Epidermal Growth Factor; ISEs: Intronic splicing enhancers; ISSs: Intronic splicing silencers; PET: Florbetapir position emission tomography; SNP: Single nucleotide polymorphism; SRE: Splicing regulatory element

\section{Acknowledgments}

Data used in the preparation of this article were obtained from the Alzheimer's Disease Neuroimaging Initiative (ADNI) database (adni.loni.usc.edu). As such, the investigators within the ADNI contributed to the design and implementation of ADNI and/or provided data but did not participate in analysis or writing of this report. A complete listing of ADNI investigators can be found at http://adni.loni. usc.edu/wp-content/uploads/how to apply/ADNI_Acknowledgement_List.pdf. Data collection and sharing for this project was funded by the Alzheimer's Disease Neuroimaging Initiative (ADNI) (National Institutes of Health Grant U01 AG024904) and DOD ADNI (Department of Defense award number W81XWH-12-2-0012). ADNI is funded by the National Institute on Aging, the National Institute of Biomedical Imaging and Bioengineering, and through generous contributions from the following: Alzheimer's Association; Alzheimer's Drug Discovery Foundation; BioClinica, Inc.; Biogen Idec Inc.; Bristol-Myers Squibb Company; Eisai Inc.; Elan Pharmaceuticals, Inc.; Eli Lilly and Company; F. Hoffmann-La Roche Ltd. and its affiliated company Genentech, Inc.; GE Healthcare; Innogenetics, N.V.; IXICO Ltd.; Janssen Alzheimer Immunotherapy Research \& Development, LLC.; Johnson \& Johnson Pharmaceutical Research \& Development LLC.; Medpace, Inc.; Merck \& Co., Inc.; Meso Scale Diagnostics, LLC.; NeuroRx Research; Novartis Pharmaceuticals Corporation; Pfizer Inc.; Piramal Imaging; Servier; Synarc Inc.; and Takeda Pharmaceutical Company. The Canadian Institutes of Health Research is providing funds to support ADNI clinical sites in Canada. Private sector contributions are facilitated by the Foundation for the National Institutes of Health (www.fnih.org). The grantee organization is the Northern California Institute for Research and Education, and the study is coordinated by the Alzheimer's Disease Cooperative Study at the University of California, San Diego. ADNI data are disseminated by the Laboratory for Neuro Imaging at the University of Southern California. Samples from the National Cell Repository for AD (NCRAD), which receives government support under a cooperative agreement grant (U24 AG21886) awarded by the National Institute on Aging (AIG), were used in this study. The support and resources from the Center for High-Performance Computing and Vice President's Clinical and Translational Research Scholar Program at the University of Utah are gratefully acknowledged.

\section{Funding}

Additional support for data analysis was provided by grant 2-4570.5 of the Swiss National Science Foundation, NLM R01 LM012535, NIA R03 AG054936, NIA R01 AG19771, NIA P30 AG10133, NLM R01 LM011360, NSF IIS-1117335, DOD W81XWH-14-2-0151, NCAA 14132004, NIGMS P50GM115318, NCATS UL1 TR001108, NIA K01 AG049050, the Alzheimer's Association, the Indiana Clinical and Translational Science Institute, and the IU Health-IU School of Medicine Strategic Neuroscience Research Initiative. The publication of this article was sponsored by the grant, NLM R01 LM012535.

\section{Availability of data and materials}

Demographic information, raw neuroimaging scan data, APOE and genomewide genotyping data, RNA-Seq data of hippocampal tissues, and diagnostic information are available from the ADNI (http://www.loni.usc.edu/ADNI/) and the Allen Brain Atlas (http://www.brain-map.org/) data repositories.

\section{About this supplement}

This article has been published as part of BMC Medical Genomics Volume 12 Supplement 1, 2019: Selected articles from the International Conference on Intelligent Biology and Medicine (ICIBM) 2018: medical genomics. The full contents of the supplement are available online at https://bmcmedgeno mics.biomedcentral.com/articles/supplements/volume-12-supplement-1.

\section{Authors' contributions}

All authors contributed substantively to this work. SH, DK, YL, and KN were involved in study conception and design. SH, JEM, SB, DK, SLR, AJS, YL, and $\mathrm{KN}$ were involved in data organization and statistical analyses. SH, $\mathrm{YL}$, and $\mathrm{KN}$ drafted the report and prepared all figures and tables. All authors were 
involved in reviewing and editing of the manuscript and approved it. All of the authors have read and approved the final manuscript.

\section{Ethics approval and consent to participate}

Written informed consent was obtained at the time of enrollment for imaging and genetic sample collection and protocols of consent forms were approved by each participating sites' Institutional Review Board (IRB).

\section{Consent for publication}

Not applicable.

\section{Competing interests}

The authors declare that they have no competing interests.

\section{Publisher's Note}

Springer Nature remains neutral with regard to jurisdictional claims in published maps and institutional affiliations.

\section{Author details}

${ }^{1}$ Department of Biomedical Informatics, University of Utah School of Medicine, Salt Lake City, UT, USA. ${ }^{2}$ Biomedical and Translational Informatics Institute, Geisinger Health System, Danville, PA, USA. ${ }^{3}$ The Huck Institutes of the Life Sciences, Pennsylvania State University, University Park, PA, USA ${ }^{4}$ Center for Neuroimaging, Department of Radiology and Imaging Sciences and Indiana Alzheimer Disease Center, Indiana University School of Medicine, Indianapolis, IN, USA. ${ }^{5}$ Center for Computational Biology and Bioinformatics, Indiana University School of Medicine, Indianapolis, IN, USA.

\section{Published: 31 January 2019}

\section{References}

1. Perl DP. Neuropathology of Alzheimer's disease. Mt Sinai J Med. 2010; 77(1):32-42.

2. Gatz M, Pedersen NL, Berg S, Johansson B, Johansson K, Mortimer JA, Posner SF, Viitanen M, Winblad B, Ahlbom A. Heritability for Alzheimer's disease: the study of dementia in Swedish twins. J Gerontol A Biol Sci Med Sci. 1997;52(2):M117-25.

3. Lambert JC, Ibrahim-Verbaas CA, Harold D, Naj AC, Sims R, Bellenguez C, DeStafano AL, Bis JC, Beecham GW, Grenier-Boley B, et al. Meta-analysis of 74,046 individuals identifies 11 new susceptibility loci for Alzheimer's disease. Nat Genet. 2013;45(12):1452-8.

4. Twine NA, Janitz K, Wilkins MR, Janitz M. Whole transcriptome sequencing reveals gene expression and splicing differences in brain regions affected by Alzheimer's disease. PLoS One. 2011;6(1):e16266.

5. Sekar S, McDonald J, Cuyugan L, Aldrich J, Kurdoglu A, Adkins J, Serrano G, Beach TG, Craig DW, Valla J, et al. Alzheimer's disease is associated with altered expression of genes involved in immune response and mitochondrial processes in astrocytes. Neurobiol Aging. 2015;36(2):583-91.

6. Sutherland GT, Janitz M, Kril JJ. Understanding the pathogenesis of Alzheimer's disease: will RNA-Seq realize the promise of transcriptomics? J Neurochem. 2011:116(6):937-46.

7. Pereira AC, Gray JD, Kogan JF, Davidson RL, Rubin TG, Okamoto M, Morrison JH, McEwen BS. Age and Alzheimer's disease gene expression profiles reversed by the glutamate modulator riluzole. Mol Psychiatry. 2017:22(2):296-305

8. Ciryam P, Kundra R, Freer R, Morimoto Rl, Dobson CM, Vendruscolo M. A transcriptional signature of Alzheimer's disease is associated with a metastable subproteome at risk for aggregation. Proc Natl Acad Sci U S A. 2016;113(17):4753-8

9. Vuong CK, Black DL, Zheng S. The neurogenetics of alternative splicing. Nat Rev Neurosci. 2016;17(5):265-81.

10. Love JE, Hayden EJ, Rohn TT. Alternative Splicing in Alzheimer's Disease. J Parkinsons Dis Alzheimers Dis. 2015;2(2):6.

11. Lai MK, Esiri MM, Tan MG. Genome-wide profiling of alternative splicing in Alzheimer's disease. Genom Data. 2014;2:290-2.

12. Tollervey JR, Wang Z, Hortobagyi T, Witten JT, Zarnack K, Kayikci M, Clark TA, Schweitzer AC, Rot G, Curk T, et al. Analysis of alternative splicing associated with aging and neurodegeneration in the human brain. Genome Res. 2011;21(10):1572-82.
13. Szymanski M, Wang R, Bassett SS, Avramopoulos D. Alzheimer's risk variants in the clusterin gene are associated with alternative splicing. Transl Psychiatry. 2011;1(7):e18

14. Malik M, Simpson JF, Parikh I, Wilfred BR, Fardo DW, Nelson PT, Estus S. CD33 Alzheimer's risk-altering polymorphism, CD33 expression, and exon 2 splicing. J Neurosci. 2013;33(33):13320-5.

15. Wang ET, Sandberg R, Luo S, Khrebtukova I, Zhang L, Mayr C, Kingsmore SF, Schroth GP, Burge CB. Alternative isoform regulation in human tissue transcriptomes. Nature. 2008;456(7221):470-6.

16. Mills JD, Nalpathamkalam T, Jacobs HI, Janitz C, Merico D, Hu P, Janitz M. RNA-Seq analysis of the parietal cortex in Alzheimer's disease reveals alternatively spliced isoforms related to lipid metabolism. Neurosci Lett. 2013:536:90-5.

17. De Jonghe $C$, Cruts $M$, Rogaeva EA, Tysoe C, Singleton A, Vanderstichele $H$, Meschino W, Dermaut B, Vanderhoeven I, Backhovens H, et al. Aberrant splicing in the presenilin-1 intron 4 mutation causes presenile Alzheimer's disease by increased Abeta42 secretion. Hum Mol Genet. 1999:8(8):1529-40.

18. Andreadis A, Brown WM, Kosik KS. Structure and novel exons of the human tau gene. Biochemistry. 1992;31(43):10626-33.

19. Goedert M, Spillantini MG, Jakes R, Rutherford D, Crowther RA. Multiple isoforms of human microtubule-associated protein tau: sequences and localization in neurofibrillary tangles of Alzheimer's disease. Neuron. 1989; 3(4):519-26.

20. Karambataki M, Malousi A, Kouidou S. Risk-associated coding synonymous SNPs in type 2 diabetes and neurodegenerative diseases: genetic silence and the underrated association with splicing regulation and epigenetics. Mutat Res. 2014;770:85-93.

21. Graveley BR. Sorting out the complexity of SR protein functions. RNA. 2000; 6(9):1197-211

22. Pagenstecher $C$, Wehner $M$, Friedl W, Rahner N, Aretz S, Friedrichs N, Sengteller M, Henn W, Buettner R, Propping P, et al. Aberrant splicing in MLH1 and MSH2 due to exonic and intronic variants. Hum Genet. 2006; 119(1-2):9-22

23. Liu HX, Cartegni L, Zhang MQ, Krainer AR. A mechanism for exon skipping caused by nonsense or missense mutations in BRCA1 and other genes. Nat Genet. 2001:27(1):55-8.

24. Faustino NA, Cooper TA. Pre-mRNA splicing and human disease. Genes Dev. 2003:17(4):419-37.

25. Han S, Jung H, Lee K, Kim H, Kim S. Genome wide discovery of genetic variants affecting alternative splicing patterns in human using bioinformatics method Genes Genom. 2017:39(4):453-9.

26. Gamazon ER, Konkashbaev A, Derks EM, Cox NJ, Lee Y. Evidence of selection on splicing-associated loci in human populations and relevance to disease loci mapping. Sci Rep. 2017;7(1):5980.

27. Lee Y, Gamazon ER, Rebman E, Lee Y, Lee S, Dolan ME, Cox NJ, Lussier YA Variants affecting exon skipping contribute to complex traits. PLoS Genet. 2012:8(10):e1002998.

28. Sonnen JA, Larson EB, Haneuse S, Woltjer R, Li G, Crane PK, Craft S, Montine TJ. Neuropathology in the adult changes in thought study: a review. J Alzheimers Dis. 2009;18(3):703-11

29. Montine TJ, Sonnen JA, Montine KS, Crane PK, Larson EB. Adult changes in thought study: dementia is an individually varying convergent syndrome with prevalent clinically silent diseases that may be modified by some commonly used therapeutics. Curr Alzheimer Res. 2012;9(6):718-23.

30. Anders S, Pyl PT, Huber W. HTSeq--a Python framework to work with highthroughput sequencing data. Bioinformatics. 2015;31(2):166-9.

31. Anders $S$, Reyes A, Huber W. Detecting differential usage of exons from RNA-seq data. Genome Res. 2012:22(10):2008-17.

32. Benson DA, Karsch-Mizrachi I, Lipman DJ, Ostell J, Wheeler DL. GenBank: update. Nucleic Acids Res. 2004:32(Database issue):D23-6.

33. Hubbard T, Barker D, Birney E, Cameron G, Chen Y, Clark L, Cox T, Cuff J, Curwen $\mathrm{V}$, Down $\mathrm{T}$, et al. The Ensembl genome database project. Nucleic Acids Res. 2002;30(1):38-41.

34. Thierry-Mieg D, Thierry-Mieg J. AceView: a comprehensive cDNA-supported gene and transcripts annotation. Genome Biol. 2006;7(Suppl 1):S12.

35. Hsu F, Kent WJ, Clawson H, Kuhn RM, Diekhans M, Haussler D. The UCSC known genes. Bioinformatics. 2006;22(9):1036-46.

36. Kallberg M, Margaryan G, Wang S, Ma J, Xu J. RaptorX server: a resource for template-based protein structure modeling. Methods Mol Biol. 2014;1137:17-27.

37. Ramanan VK, Risacher SL, Nho K, Kim S, Swaminathan S, Shen L, Foroud TM, Hakonarson $\mathrm{H}$, Huentelman MJ, Aisen PS, et al. APOE and BCHE as 
modulators of cerebral amyloid deposition: a florbetapir PET genome-wide association study. Mol Psychiatry. 2014;19(3):351-7.

38. Nho K, Corneveaux JJ, Kim S, Lin H, Risacher SL, Shen L, Swaminathan S, Ramanan VK, Liu Y, Foroud T, et al. Identification of functional variants from whole-exome sequencing, combined with neuroimaging genetics. Mol Psychiatry. 2013;18(7):739.

39. Nho K, Corneveaux JJ, Kim S, Lin H, Risacher SL, Shen L, Swaminathan S, Ramanan VK, Liu Y, Foroud T, et al. Whole-exome sequencing and imaging genetics identify functional variants for rate of change in hippocampal volume in mild cognitive impairment. Mol Psychiatry. 2013;18(7):781-7.

40. Nho K, Kim S, Risacher SL, Shen L, Corneveaux JJ, Swaminathan S, Lin H, Ramanan VK, Liu Y, Foroud TM, et al. Protective variant for hippocampal atrophy identified by whole exome sequencing. Ann Neurol. 2015;77(3):547-52.

41. Ramanan VK, Risacher SL, Nho K, Kim S, Shen L, McDonald BC, Yoder KK, Hutchins GD, West JD, Tallman EF, et al. GWAS of longitudinal amyloid accumulation on 18F-florbetapir PET in Alzheimer's disease implicates microglial activation gene IL1RAP. Brain. 2015;138(Pt 10):3076-88.

42. Pujadas L, Rossi D, Andres R, Teixeira CM, Serra-Vidal B, Parcerisas A, Maldonado R, Giralt E, Carulla N, Soriano E. Reelin delays amyloid-beta fibril formation and rescues cognitive deficits in a model of Alzheimer's disease. Nat Commun. 2014:5:3443.

43. Herring A, Donath A, Steiner KM, Widera MP, Hamzehian S, Kanakis D, Kolble K, EIAli A, Hermann DM, Paulus W, et al. Reelin depletion is an early phenomenon of Alzheimer's pathology. J Alzheimers Dis. 2012;30(4):963-79.

44. Seripa D, Matera MG, Franceschi M, Daniele A, Bizzarro A, Rinaldi M, Panza F, Fazio VM, Gravina C, D'Onofrio G, et al. The RELN locus in Alzheimer's disease. J Alzheimers Dis. 2008;14(3):335-44.

45. Reif A, Grunblatt E, Herterich S, Wichart I, Rainer MK, Jungwirth S, Danielczyk W, Deckert J, Tragl KH, Riederer P, et al. Association of a functional NOS1 promoter repeat with Alzheimer's disease in the VITA cohort. J Alzheimers Dis. 2011;23(2):327-33.

46. Folsom TD, Fatemi SH. The involvement of Reelin in neurodevelopmental disorders. Neuropharmacology. 2013;68:122-35.

47. Feher A, Juhasz A, Pakaski M, Kalman J, Janka Z. Genetic analysis of the RELN gene: gender specific association with Alzheimer's disease. Psychiatry Res. 2015;230(2):716-8.

48. Kocherhans S, Madhusudan A, Doehner J, Breu KS, Nitsch RM, Fritschy JM, Knuesel I. Reduced Reelin expression accelerates amyloid-beta plaque formation and tau pathology in transgenic Alzheimer's disease mice. $J$ Neurosci. 2010;30(27):9228-40.

49. Chen N, Bao Y, Xue Y, Sun Y, Hu D, Meng S, Lu L, Shi J. Meta-analyses of RELN variants in neuropsychiatric disorders. Behav Brain Res. 2017;332:110-9.

50. Bothwell M, Giniger E. Alzheimer's disease: neurodevelopment converges with neurodegeneration. Cell. 2000;102(3):271-3.

51. Zhang $\mathrm{YH}$, Jin $\mathrm{CZ}$, Jang JH, Wang Y. Molecular mechanisms of neuronal nitric oxide synthase in cardiac function and pathophysiology. J Physiol. 2014;592(15):3189-200.

52. Lukiw WJ, Rogaev El. Genetics of aggression in Alzheimer's disease (AD). Front Aging Neurosci. 2017:9:87.

53. Kim D, Shivakumar M, Han S, Sinclair MS, Lee YJ, Zheng Y, Olopade OI, Kim D, Lee Y. Population-dependent intron retention and DNA methylation in breast Cancer. Mol Cancer Res. 2018;16(3):461-9.

54. Lev Maor G, Yearim A, Ast $\mathrm{G}$. The alternative role of DNA methylation in splicing regulation. Trends Genet. 2015;31(5):274-80.

55. Gelfman S, Cohen N, Yearim A, Ast G. DNA-methylation effect on cotranscriptional splicing is dependent on GC architecture of the exonintron structure. Genome Res. 2013;23(5):789-99.

56. Merkin J, Russell C, Chen P, Burge CB. Evolutionary dynamics of gene and isoform regulation in mammalian tissues. Science. 2012;338(6114):1593-9.

57. Zhang X, Chen MH, Wu X, Kodani A, Fan J, Doan R, Ozawa M, Ma J, Yoshida $\mathrm{N}$, Reiter JF, et al. Cell-type-specific alternative splicing governs cell fate in the developing cerebral cortex. Cell. 2016;166(5):1147-62 e1115

\section{Ready to submit your research? Choose BMC and benefit from}

- fast, convenient online submission

- thorough peer review by experienced researchers in your field

- rapid publication on acceptance

- support for research data, including large and complex data types

- gold Open Access which fosters wider collaboration and increased citations

- maximum visibility for your research: over $100 \mathrm{M}$ website views per year

At BMC, research is always in progress.

Learn more biomedcentral.com/submissions 